Pacific Journal of Mathematics

ON A THEOREM OF JORDAN 


\title{
ON A THEOREM OF JORDAN
}

\author{
Marshall Hall, Jr.
}

1. Introduction. In 1872 Jordan [4] showed that a finite quadruply transitive group in which only the identity fixes four letters must be one of the following groups: the symmetric group on four or five letters, the alternating group on six letters, or the Mathieu group on eleven letters.

In this paper Jordan's theorem on quadruply transitive groups is generalized in two ways. The number of letters is not assumed to be finite; and instead of assuming that the subgroup fixing four letters consists of the identity alone, we only assume it to be a finite group of odd order. The conclusion is essentially the same as that of Jordan's theorem, the only other group satisfying the hypotheses being the alternating group on seven letters.

2. Proof of the main theorem. The theorem is the following:

THEOREM 2.1. A group $G$ quadruply transitive on a set of letters, finite or infinite, in which a subgroup $H$ fixing four letters is of finite odd order, must be one of the following groups: $S_{4}, S_{5}, A_{6}, A_{7}$ or the Nathieu group on 11 letters.

Case 1. G on not more than seven letters. A quadruply transitive group on 4 or 5 letters must be the symmetric group. On six letters its order must be at least $6 \cdot 5 \cdot 4 \cdot 3$, and hence it is $A_{6}$ or $S_{6}$. On seven letters, its index is at most 6 in $S_{7}$. As $S_{7}$ does not have a subgroup of index 3 or 6 , the only possibilities are $A_{7}$ and $S_{7}$. In both $S_{6}$ and $S_{7}$ there are elements of order two fixing at least four letters, and so these groups do not satisfy our hypothesis.

To treat the case in which $G$ is on more than seven letters, we begin with two simple lemmas.

LeMMA 2.1. Elements $a, b$ in a group, satisfying the relations

$$
a^{2}=1, b^{2}=1,(a b)^{s}=1,
$$


generate the dihedral group of order $2 s$. If $s=2 t-1$ is odd, then a power of $y=a b$ transforms $a$ into $b$. If $s=2 r$ is even, then $a$ and $b$ permute with $y^{r}$.

Proof. With $y=a b$, we have

$$
a^{2}=1, y^{s}=1, b=a y=y^{-1} a \text {. }
$$

If $s=2 t-1$, then

$$
y^{-t} a y^{t}=a y^{2 t}=b \text {. }
$$

If $s=2 r$, then

$$
a y^{r}=y^{-r} a=y^{r} a \text {. }
$$

LEMMA 2.2. If $G$ is a k-ply transitive permutation group and $P$ is a Sylow subgroup of the finite subgroup $H_{k}$ fixing $k$ letters, then $N_{G}(P)$, the normalizer in $G$ of $P$ is $k$-ply transitive on the letters fixed by $P$.

Proof. (Compare $\left[1\right.$, p. 212; 6, p. 259].) Let $a_{1}, \cdots, a_{k}$ and $b_{1}, \cdots, b_{k}$ be letters fixed by $P$. Then in $G$ there is an element $x$ taking $a_{1}, \ldots, a_{k}$ into $b_{1}, \ldots, b_{k}$. Here $x^{-1} P x=P^{\prime}$ is a Sylow subgroup of the group $H_{k}^{\prime}$ fixing $b_{1}, \ldots, b_{k}$. But $P$ also fixes $b_{1}, \ldots, b_{k}$. Thus $P$ and $P^{\prime}$ are conjugate in $H_{k}^{\prime}$, and there will be a $y$ fixing $b_{1}, \ldots, b_{k}$ with

$$
y^{-1} P^{\prime} y=P
$$

Hence $z=x y$ is an element normalizing $P$ and taking $a_{1}, \cdots, a_{k}$ into $b_{1}, \cdots, b_{k}$. Trivially $N_{G}(P)$ takes the letters fixed by $P$ into themselves. We note that $P$ need not be a Sylow subgroup of $G$.

From here on $G$ will denote, as in Theorem 2.1, a group quadruply transitive on more than seven letters, and $H$ a subgroup of odd order $m$ fixing four letters.

LEMMA 2.3. The group $G$ contains elements of order 2, and all elements of order 2 are conjugate. Either 1) every element of order 2 fixes two letters or 2) every element of order 2 fixes three letters.

Proof. By quadruple transitivity $G$ contains an element

$$
g=(12)(34) \cdots
$$

Here $g^{2}$ fixes $1,2,3,4$, and so belongs to $H$ and will be of finite odd order $m_{1}$. 
Thus

$$
x=g^{m_{1}}=(12)(34) \cdots,
$$

with $x^{2}=1$. As $H$ is of odd order, any element $u$ of order two will fix at most three letters, and hence displace at least four letters. With

$$
u=(a b)(c d) \ldots
$$

there is a conjugate of $u$,

$$
v=w^{-1} u w=(12)(34) \ldots
$$

Either $v=x$, or $v x$ fixes four letters and is of odd order, whence, by Lemma 2.1, $v$ and $x$ are conjugate. Thus all elements of order two are conjugate. On the other hand, there is in $G$ an element $z=(1)(2)(34) \ldots$, and either $z$ or an odd power of $z$ is an element of order two fixing at least two letters. Hence every element of order two fixes either two or three letters, since they fix at least two and not as many as four.

Case 2. $G$ on more than seven letters. Let

$$
a_{1}=(1)(2)(34) \cdots
$$

be an element of order two and

$$
b=(12)(34) \ldots
$$

another element of order two. Then $f=a_{1} b=(12)(3)(4) \cdots$ will be of even order, and $f^{2}$ will be of odd order $m_{1}$. Hence $f^{m_{1}}=a_{3}$ is of order two and by Lemma 2.1 will permute with $a_{1}$. Hence in $G$ we have permuting elements of order two, with $a_{2}=a_{1} a_{3}$ :

$$
\begin{aligned}
& a_{1}=(1)(2)(34) \cdots \\
& a_{2}=\left(\begin{array}{ll}
1 & 2
\end{array}\right)(34) \cdots \\
& a_{3}=\left(\begin{array}{ll}
1 & 2
\end{array}\right)(3)(4) \cdots
\end{aligned}
$$

Now $a_{2}$ as an element of order 2 fixes either two letters 5 and 6 , or three letters 5,6 , and 7. As $a_{1}$ permutes with the element $a_{2}$, it takes these letters into themselves. But $a_{1}$ fixes 1 and 2 and at most one other letter. Hence we have 


$$
\begin{array}{lll}
a_{1}=(1)(2)(34)(56) \ldots & & a_{1}=(1)(2)(34)(56)(7) \ldots \\
a_{2}=(12)(34)(5)(6) \cdots & \text { or } & a_{2}=(12)(34)(5)(6)(7) \ldots \\
a_{3}=(12)(3)(4)(56) \ldots & & a_{3}=(12)(3)(4)(56)(7) \ldots
\end{array}
$$

the first case arising if elements of order 2 all fix two letters, the second if all fix three letters. The elements $a_{1}, a_{2}, a_{3}$ of $(2.2)$ and the identity form a four group $V$. Further letters will occur in sets of four which will be sets of transitivity for $V$ :

$$
\begin{aligned}
& a_{1}=(1)(2)(34)(56)(7)(h i)(j k) \ldots \\
& a_{2}=(12)(34)(5)(6)(7)(h j)(i k) \ldots \\
& a_{3}=(12)(3)(4)(56)(7)(h k)(i j) \ldots
\end{aligned}
$$

Here it is understood that the 7 may not be present.

The order of the subgroup $K$ taking $h, i, j, k$ into themselves will be $24 m$, and $H=H(h, i, j, k)$ of order $m$ will be normal in $K$. There will be a subgroup $U, K \supset U \supset H$, in which $h, i, j, k$ are permuted in the following way:

$$
\begin{aligned}
& (h i)(j k) \\
& (h j)(i k) \\
& (h k)(i j .) \\
& (h j i k) \\
& (h k i j) \\
& (h i)(j)(k) \\
& (h)(i)(j k) .
\end{aligned}
$$

Now $U$ is of order $8 m$, and so a Sylow subgroup of $U$ will be of order 8. The elements taking $h, i, j, k$ into themselves in a particular way will be a coset of $H$ in $U$. As $H$ is normal in $U$, a group of order 8 in $U$ will have one element from each coset and be isomorphic to $H / U$ and hence faithfully represented by the permutations on these letters. $V$ will be contained in a Sylow subgroup of order 8 in $U$. This yields

$$
\begin{aligned}
& a_{1}=(1)(2)(34)(56)(7)(h i)(j k) \ldots \\
& a_{2}=(12)(34)(5)(6)(7)(h j)(i k) \ldots
\end{aligned}
$$




$$
\begin{aligned}
& a_{3}=(12)(3)(4)(56)(7)(h k)(i j) \ldots \\
& u=(1)(2)(3546) \quad(7)(h j i k) \ldots \\
& a_{1} u=(1)(2)(3645) \quad \text { (7) }(h k i j) \ldots \\
& a_{2} u=\left(\begin{array}{ll}
1 & 2
\end{array}\right)(36)(45) \quad(7)(h i)(j)(k) \ldots \\
& a_{3} u=(12)(35)(46) \quad(7)(h)(i)(j k) \ldots
\end{aligned}
$$

or the same permutations with 5 and 6 interchanged. The way in which the last four elements permute the letters $1, \cdots, 7$ is determined by the relations

$$
u^{2}=a_{1}, \quad u^{-1} a_{2} u=a_{3}, \quad\left(a_{2} u\right)^{2}=1 .
$$

Here $u$ normalizes $V$ and so fixes the only letter, 7 , fixed by $V$, if the 7 occurs. Also $u$ must take the fixed letters of $a_{3}$ into those of $a_{2}$, whence

$$
u=\left(\begin{array}{lll}
3 & 4 & \cdots \\
5 & 6 & \cdots
\end{array}\right) \quad \text { or } \quad u=\left(\begin{array}{lll}
3 & 4 & \cdots \\
6 & 5 & \cdots
\end{array}\right)
$$

but also $u^{2}=a_{1}$, whence

$$
u=(3546) \cdots \text { or } u=(3645) \cdots \text {. }
$$

Finally, $u$ must fix 1 and 2 or interchange them. But if $u$ interchanges 1 and 2, then $a_{2} u$ is of order 2 and fixes the letters $1,2, j, k$. Thus

$$
u=(1)(2)(3546) \cdots \text { or } u=(1)(2)(3645) \cdots \text {, }
$$

and the rest follows.

Each further transitive constituent of $V$ such as $h, i, j, k$ yields a group $S$ such as that in $(2.5)$. The elements

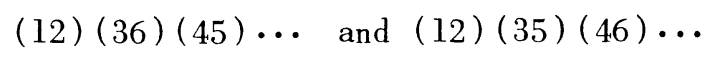

in each of these groups fix two letters of the constituent. Since an element of order two cannot fix four letters, each constituent yields a different element permuting the first six letters in the way (12) (36) (45). But there are at most $m$ elements with this effect on the first six letters. Thus if there are $t$ such constituents, $t \leq m$ is finite and $G$ is a group on $n=4 t+6$ or $4 t+7$ letters. If $G$ is on 10 or 11 letters we have $t=1$.

There is no quadruply transitive group on 10 letters (except of course $A_{10}$ and $\left.S_{10}\right)$. For the normalizer of a cycle of length 7 by Lemma 2.2 is $S_{3}$ on the 
remaining three letters; and so this normalizer, which is the subdirect product of $S_{3}$ and the normalizer on the letters of the 7-cycle, will pair a 3-cycle with the identity. Hence $G$ contains a 3-cycle, and, being quadruply transitive, all 3-cycles and so contains $A_{10}$.

On 11 letters $G$ is of order $11 \cdot 10 \cdot 9 \cdot 8 m$, and even without assuming $m$ odd, consideration of normalizers of Sylow subgroups fixing four letters shows that we must have $m=1$. The group of order 8 fixing three letters contains a single element of order $2[3, \mathrm{p} .311]$, and so is the cyclic or quaternion group. The cyclic group, having only 4 automorphisms, could not have a normalizer triply transitive on the remaining three letters, for then $G$ would contain a 3-cycle. Hence the subgroup fixing three letters must be the quaternion group $Q$. Then $G$ will be a transitive extension of $Q$, and the methods of T. C. Holyoke [3] readily enable us to construct from $Q$ not only the quadruply transitive Mathieu group on 11 letters, but the quintuply transitive group on 12 letters.

We shall now show that $t>1$ conflicts with the hypothesis that $H$ is of odd order, and thus complete the proof of our theorem. If $w, x, y, z$ is another transitive constituent of $V$, we have

$$
a_{2} u=(12)(36)(45)(7)(h i)(j)(k) \cdots
$$

from (2.5) and will have another element

$$
a_{2} u^{\prime}=(12)(36)(45)(7)(w x)(y)(z) \cdots
$$

Each of these elements permutes with $a_{1}$ and transforms $a_{2}$ into $a_{3}$ and $a_{3}$ into $a_{2}$. Their product is an element $q$ fixing the first six (or seven) letters and so of odd order. Also $q$ centralizes $V$. By Lemma 2.1 a power of $q$ transforms $a_{2} u$ into $a_{2} u^{\prime}$, and so takes the fixed letters $j, k$ of $a_{2} u$ into the fixed letters $y, z$ of $a_{2} u^{\prime}$. Centralizing $V$, this element must take the entire constituent $h i j k$ into $w x y z$. Hence there is a group $C$ in $G$ which fixes the first six (or seven) letters, centralizes $V$, and is transitive on the $t$ remaining constituents of $V$. An element of $C$ taking a constituent of $V$ into itself, being of odd order, must fix all four letters. Thus the transitive constituents of $C$ are (1) (2) (3) (4) (5) (6) (7) $T_{h}, T_{i}, T_{j}, T_{k}$, the last four sets of $t$ each, the letters $h, i, j, k$ being in different constituents of $C$.

Let $p$ be a prime dividing $t$. (Here we use the assumption $t>1$.) Let $P$ be the corresponding Sylow subgroup of $C$. Then $P$ displaces all $4 t$ letters which $C$ displaces, since a subgroup of $C$ fixing a letter is of index $t \equiv 0(\bmod p)$ and cannot contain such a Sylow subgroup. Now let $P_{1}$ be a Sylow subgroup of $H$, 
the subgroup fixing $1,2,3,4$, which contains $P$. Then $P_{1}$ displaces the $4 t$ letters of $C$ and no others, unless possibly we have the case

$$
p=3, t=3^{w}, n=4 t+7
$$

where $P_{1}$ might be on $4 t+3$ letters. This possibility will be considered later. With $P_{1}$ on $4 t$ letters, by Lemma 2.2 , the group $N_{G}\left(P_{1}\right)$ is quadruply transitive on the first six or seven letters and so contains $A_{6}$ or $A_{7}$ on these letters. But the subgroup taking the first six (or seven) letters into themselves also contains the element $u$ of (2.5) which is not in the alternating group on these letters. Thus in $G$ we have the full symmetric group on the first six or seven letters and hence some element fixing the first four letters and interchanging the fifth and sixth. This conflicts with the hypothesis that $H$ is of odd order. Finally, consider the possibility that

$$
t=3^{w}, n=4 t+7
$$

and that $P_{1}$ displaces $5,6,7$ as well as the $4 t$ letters of $P$. If $w>1$, then surely $5,6,7$ are a transitive constituent of $P_{1}$ and there is an element

$$
z=(1)(2)(3)(4)(567) \ldots
$$

in $G$. If $w=1$, then $P$ is of order 3 , and even if, in $P_{1}, 5,6,7$ are in a constituent with $8,9,10$ and $11,12,13$ of $P$, since there is an element (5) (6) (7) $(8,9,10)(11,12,13)$, there will also be one such as $z$ fixing $8,9,10$. But with

$$
z=(1)(2)(3)(4)(567) \cdots
$$

and $u$ of $(2.5)$ we have

$$
(z u)^{3}=(1)(2)(35)(4)(6)(7) \cdots,
$$

contradicting the assumption that a subgroup $H$ fixing 4 letters is of odd order.

\section{REFERENCES}

1. W. Burnside, The theory of groups, Cambridge University Press, second edition 1911.

2. R. D. Carmichael, The ory of groups of finite order, Ginn and Co., Boston, 1937.

3. T. C. Holyoke, On the structure of multiply transitive permutation groups, Amer. J. Math. 74 (1952), 787 - 796. 
4. C. Jordan, Recherches sur les substitutions, J. Math. Pures Appl. (2) 17 (1872), $351-363$.

5. G. A. Miller, Limits of the degree of transitivity of substitution groups, Bull. Amer. Math. Soc. 22 (1915), $68-71$.

6. E. Witt, Die 5-fach transitiven Gruppen von Mathieu, Abh. Math. Sem. Univ. Hamburg 12 (1938), $256-264$.

The Ohio State University 


\section{PACIFIC JOURNAL OF MATHEMATICS}

\section{EDITORS}

\author{
M.M. SCHIFFE R* \\ Stanford University \\ Stanford, California \\ E. HEWITT \\ University of Washington \\ Seattle 5, Washington
}

\author{
R.P. DILWORTH \\ Calif ornia Institute of Technology \\ Pasadena 4, California \\ E. F. BECKENBACH** \\ University of California \\ Los Angeles 24, California
}

\section{ASSOCIATE EDITORS}

$\begin{array}{llll}\text { H. BUSEMANN } & \text { P.R. HALMOS } & \text { BØRGE JESSEN } & \text { J. J. STOKER } \\ \text { HERBERT FEDERER } & \text { HEINZ HOPF } & \text { PAUL LÉVY } & \text { E. G. STRAUS } \\ \text { MARSHALL HALL } & \text { R.D. JAMES } & \text { GEORGE PÓLYA } & \text { KÔSAKU YOSIDA }\end{array}$

\section{SPONSORS}

UNIVERSITY OF BRITISH COLUMBIA

UNIVERSITY OF SOUTHERN CALIFORNIA

CALIFORNIA INSTITUTE OF TECHNOLOGY

UNIVERSITY OF CALIFORNIA, BERKELEY

STANFORD RESEARCH INSTITUTE

UNIVERSITY OF CALIFORNIA, DAVIS

STANFORD UNIVERSITY

UNIVERSITY OF CALIFORNIA, LOS ANGELES

WASHINGTON STATE COLLEGE

UNIVERSITY OF CALIFORNIA, SANTA BARBARA

UNIVERSITY OF WASHINGTON

UNIVERSITY OF NEVADA

OREGON STATE COLLEGE

AMERICAN MATHEMATICAL SOCIETY

UNIVERSITY OF OREGON

HUGHES AIRCRAFT COMPANY

Mathematical papers intended for publication in the Pacific Journal of Mathematics should be typewritten (double spaced), and the author should keep a complete copy. Manuscripts may be sent to any of the editors. Manuscripts intended for the outgoing editors should be sent to their successors. All other communications to the editors should be addressed to the managing editor, E.G. Straus, at the University of California Los Angeles 24, California.

50 reprints of each article are furnished free of charge; additional copies may be obtained at cost in multiples of 50 .

The Pacific Journal of Mathematics is published quarterly, in March, June, September, and December. The price per volume (4 numbers) is $\$ 12.00$; single issues, $\$ 3.50$; back numbers (Volumes $1,2,3$ ) are available at $\$ 2.50$ per copy. Special price to individual faculty members of supporting institutions and to individual members of the American Mathematical Society: $\$ 4.00$ per volume; single issues, $\$ 1.25$.

Subscriptions, orders for back numbers, and changes of address should be sent to the publishers, University of California Press, Berkeley 4, California.

Printed at Ann Arbor, Michigan. Entered as second class matter at the Post Office, Berkeley, California.

* To be succeeded in 1955, by H.L. Royden, Stanford University, Stanford, California.

** To be succeeded in 1955, by E.G. Straus, University of California, Los Angeles 24, Calif.

UNIVERSITY OF CALIFORNIA PRESS - BERKELEY AND LOS ANGELES

COPYRIGHT 1954 BY PACIFIC JOURNAL OF MATHEMATICS 


\section{Pacific Journal of Mathematics}

\section{Vol. 4, No. $2 \quad$ June, 1954}

Henry Ludwig Alder, Generalizations of the Rogers-Ramanujan

identities ........................................ 161

E. M. Beelsey, Concerning total differentiability of functions of class $P$. . . 169

L. Carlitz, The number of solutions of some special equations in a finite

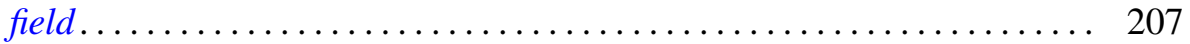

Marshall Hall, On a theorem of Jordan ............................ 219

J. D. Hill, Remarks on the Borel property..................... 227

Joseph Lehner, Note on the Schwarz triangle functions ............... 243

Arthur Eugene Livingston, A generalization of an inequality due to

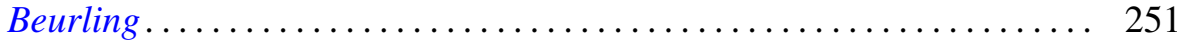

Edgar Reich, An inequality for subordinate analytic functions .......... 259

Dan Robert Scholz, Some minimum problems in the theory of functions ... . 275

J. C. Shepherdson, On two problems of Kurepa ................. 301

Abraham Wald, Congruent imbedding in F-metric spaces ............ 305

Gordon L. Walker, Fermat's theorem for algebras ............... 317 